\begin{tabular}{|c|c|c|}
\hline Beitr. Ent. & Keltern & ISSN 0005-805X \\
\hline $\mathbf{5 7}(2007) 2$ & S. $401-418$ & 21.12 .2007 \\
\hline
\end{tabular}

\title{
RICHARD KORSCHEFSKY (1902-1946) und seine grundlegenden Arbeiten über Coccinellidae und die Larven der Coleoptera*
}

Mit 16 Figuren und 2 Tabellen

Bernhard Klausnitzer

* Nach einem Vortrag anlässlich der Feier zum 120. Jahrestag des Bestehens des Deutschen Entomologischen Instituts am 28.06.2006 in Müncheberg.

\section{Zusammenfassung}

Es wird eine Übersicht über den Lebenslauf von Richard KoRsCHEFSKY gegeben und mit Fotos illustriert, die vor allem seine Tätigkeit am Deutschen Entomologischen Institut betreffen. Seine besonderen Verdienste bei der Erforschung der Coleoptera: Coccinellidae werden vorgestellt. Er bearbeitete die Coccinellidae in zwei Lieferungen des Junk-SchenkLInG-Katalogs (659 Seiten, 3279 Arten). Als Begründer einer Reihe von Bestimmungstabellen über Larven der Coleoptera hat er mit fünf Beiträgen wesentlich zu deren Kenntnis beigetragen. Ein Verzeichnis der Schriften von RichARD KORSCHEFSKY wird vorgelegt.

\section{Summary}

An overview of the life of Richard Korschefsky is presented, illustrated with photos. His work at the Deutsches Entomologisches Institut is given particular attention and his remarkable achievements in research on the Coleoptera: Coccinellidae reviewed. He treated the Coccinellidae in two parts of the JunkSchenkLing Catalogue (659 pages, 3279 species). As originator of a series of identification keys to the larvae of Coleoptera, he made five contributions which significantly improved our knowledge on these. A list of the publications of RichaRD KORSCHEFSKY is presented.

\section{Einleitung}

RICHARD Korschefsky ist mir bei meinen entomologischen Arbeiten immer wieder begegnet. Vor allem seine Arbeiten über Käferlarven und seine diesbezügliche Sammlung waren und sind ganz wesentliche Grundlagen für meine eigenen Arbeiten über die Larven der Coleoptera. Aber auch beim Studium der Coccinellidae stieß ich auf Richard Korschefsky, so dass mein Interesse an diesem Entomologen bald geweckt wurde.

Sein früher Tod in einer Zeit, als die Entomologie in Deutschland ganz am Boden lag, hat sicher dazu beigetragen, dass Richard Korschefsky zwar durch seine Publikationen viel zitiert, als Persönlichkeit jedoch kaum in das allgemeine Wissen aufgenommen wurde (erst 1963 erschien ein Nachruf aus der Feder seines früheren Kollegen GüNther SchmidT).

Die Feier zum 120. Geburtstag des Deutschen Entomologischen Instituts ist willkommener Anlass, an diesen bedeutenden Forscher zu erinnern und sein wissenschaftliches Werk aus heutiger Sicht zu betrachten, ist er doch ein früherer Kollege der heutigen Belegschaft und hat 18 Jahre dazu beigetragen, den Ruf des Instituts weltweit zu erhalten und zu vermehren. 


\section{Wer war Richard KorschefsKY?}

Richard Korschefsky (Fig. 1) wurde am 17.09.1902 in Wittenberge als fünftes Kind des Kaufmannes Albert Korschefsky geboren. Dort besuchte er die Städtische Realschule bis zur Tertia. Am 01.01.1918 begann er eine Lehre in der Naturalien- und Lehrmittelanstalt ERNST A. Bötтcher, Berlin, wo er bis 1927 als Gehilfe tätig war. In dieser Zeit war er sehr bemüht, seine Bildung zu vervollkommnen, u. a. absolvierte er eine private Weiterbildung, um das Abitur nachzuholen.

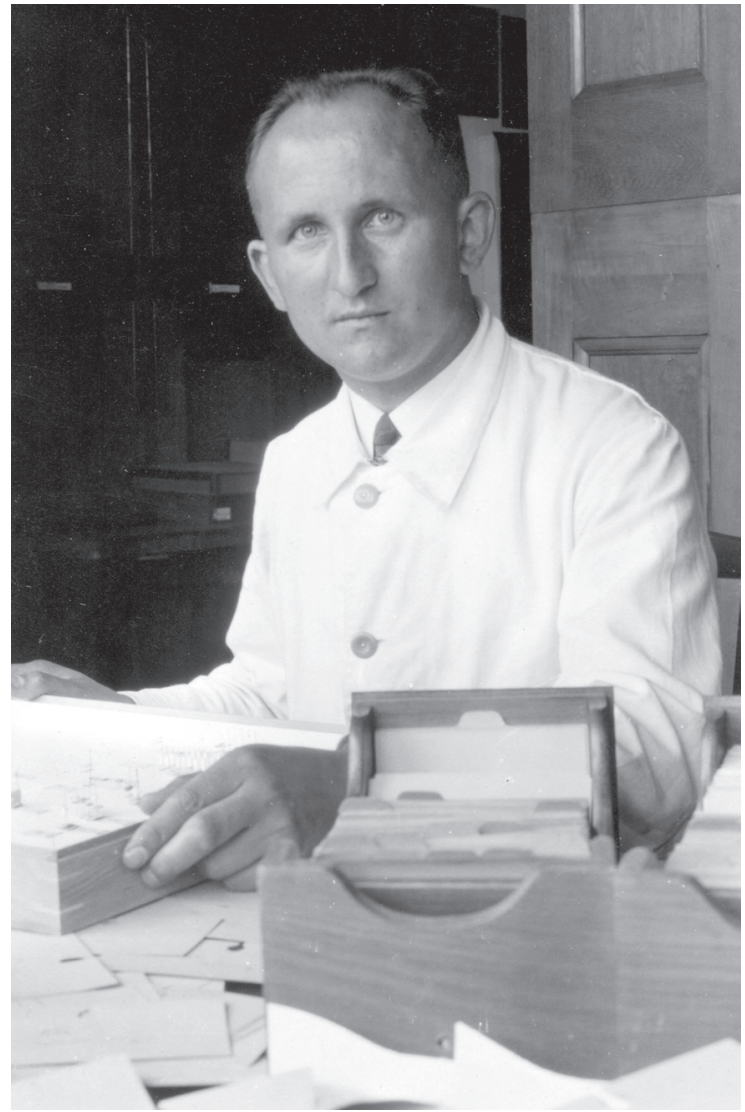

Fig. 1: Richard Korschefsky im Jahre 1932 (Messter, Berlin, Kino-Optik-Photo) (Portraitsammlung 2180).

Auf diese Weise erschloss sich ihm eine Lebensstellung: vom 01.07.1927 an war er zunächst Präparator am Deutschen Entomologischen Institut, zuletzt arbeitete Richard Korschefsky als technischer Inspektor (Fig. 2). Auch während der Anstellung am Deutschen Entomologischen Institut ließ sein Bildungswille nicht nach. Er erreichte am 10.04.1934 die Universitätsreife durch Begabtenförderung. WALTHER HoRN bewilligt ihm in einem Schreiben vom 20.04.1934 die notwendige Zeit für ein Universitätsstudium „und zwar wird Herr Korschefsky bis zur Hälfte seiner Dienststunden befreit werden". Auf diesem Schreiben findet sich ein handschriftlicher Vermerk: „musste aus Zeitmangel die bereits vollzogene Immatrikulation im Mai 1934 rückgängig machen, darum dieses $S c h r e i-$ ben zurück. Korschefsky. Juli 1936“.

Vom 1937-1944 war Richard KorSCHEFSKY ehrenamtlich als Schriftleiter der „Entomologischen Blätter“ tätig. Er gab die Bände 33 bis 44 heraus, das sind 8 Bände mit 2098 Seiten (Evers 1974), außerdem ein Sachverzeichnis für die Jahrgänge 1 bis 37 (1905-1941), 112 Seiten.

Das Deutsche Entomologische Institut war wegen seiner umfassenden Aufgaben auf dem Gebiet des Pflanzenschutzes (Diagnose, Prognose und Empfehlungen für die Praxis) als „Wehrwirtschaftsbetrieb“ eingestuft worden. So war es möglich, dass Richard Korschefsky auf Antrag von Hans Sachtleben „Uk.“ (unabkömmlich) gestellt wurde. Auf KorschefsKys eigenen Wunsch (Brief von SachtLeben an die Generalverwaltung der Kaiser-Wilhelm-Gesellschaft vom 16.08.1944) wurde dieser Status aufgehoben, und es kam zur Einberufung am 23.03.1945.

Richard Korschefsky starb im Juli 1946 in sowjetischer Kriegsgefangenschaft (Lazarett in Nowohrad-Wolinskij, Shytomyrska Oblast, Ukraine). Ein genaues Todesdatum ist nicht bekannt. Er wurde am 30.09.1946 vom Amtsgericht Tempelhof für tot erklärt. 
Richard Korschefsky war mit Anneliese Korschefsky, geb. Bruns verheiratet. Ob er Kinder hatte, konnte der Verfasser nicht in Erfahrung bringen.

Die Veranstaltung zum 120. Geburtstag des Deutschen Entomologischen Instituts findet im wahrscheinlich 60. Jahr nach seinem Tode statt, und wir wissen nicht einmal, wann er starb.

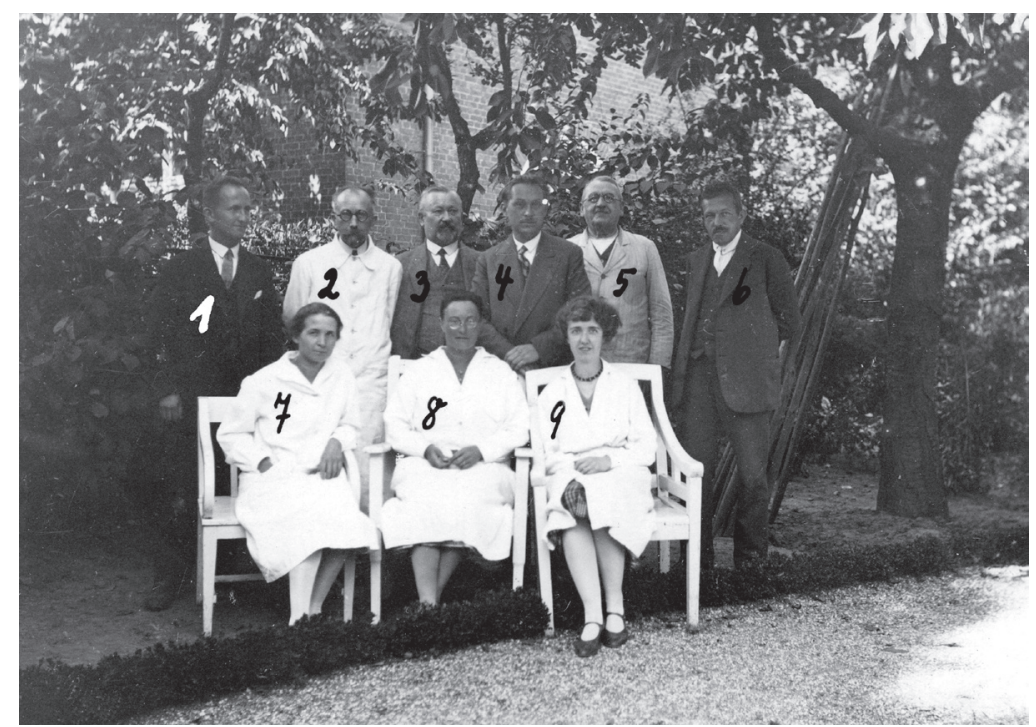

Fig. 2: Mitarbeiter des Deutschen Entomologischen Instituts 1929. 1 = Richard KorsChefsKY, 2 = WALther Horn, 3 = S. Schenkling, $4=$ H. John, $5=$ G. Lettau, $6=$ Erich Schmidt, $7=$ E. Oelze, $8=$ Chr. Doering, 9 = I. KAHLE (GR 114).

\section{Richard Korschefsky und das Deutsche Entomologische Institut}

Richard Korschefskys Verbindung zum Deutschen Entomologischen Institut ist sehr eng gewesen. Der geniale Walther Horn hat ihn gefördert und ermöglicht, dass er seine Begabung voll entfalten konnte.

Richard Korschefsky hat diese Großzügigkeit nie vergessen. Das Institut war sein Leben. Man spürt das aus seinem Artikel zum 50-jährigen Jubiläum (Fig. 3), aber auch aus dem Nachruf für Walther Horn. Er schrieb 1936:89 in dem erwähnten Artikel über das DEI: „... nichts ist dem Institut erspart geblieben. Menschliche Niedertracht und Mißgunst, wirtschaftliche Not und höhere Gewalt haben seine Existenz mehrfach in Frage gestellt, und doch immer wieder hat es sich bewähren können, hat es den Kampf mit seinen Widersachern aufgenommen und ist siegreich daraus hervorgegangen."

Am Schluss (S. 92) heißt es: „Es ist wohl anzunehmen, daß sich die gesamte entomologische Wissenschaft in dem Wunsche einig sein wird, daß das Deutsche Entomologische Institut in gleicher segensreicher Weise wie bisher noch auf lange Zeit hin wirken möge!". Diesem Wunsch ist nichts hinzuzufügen, er bedarf ständiger Bekräftigung. 


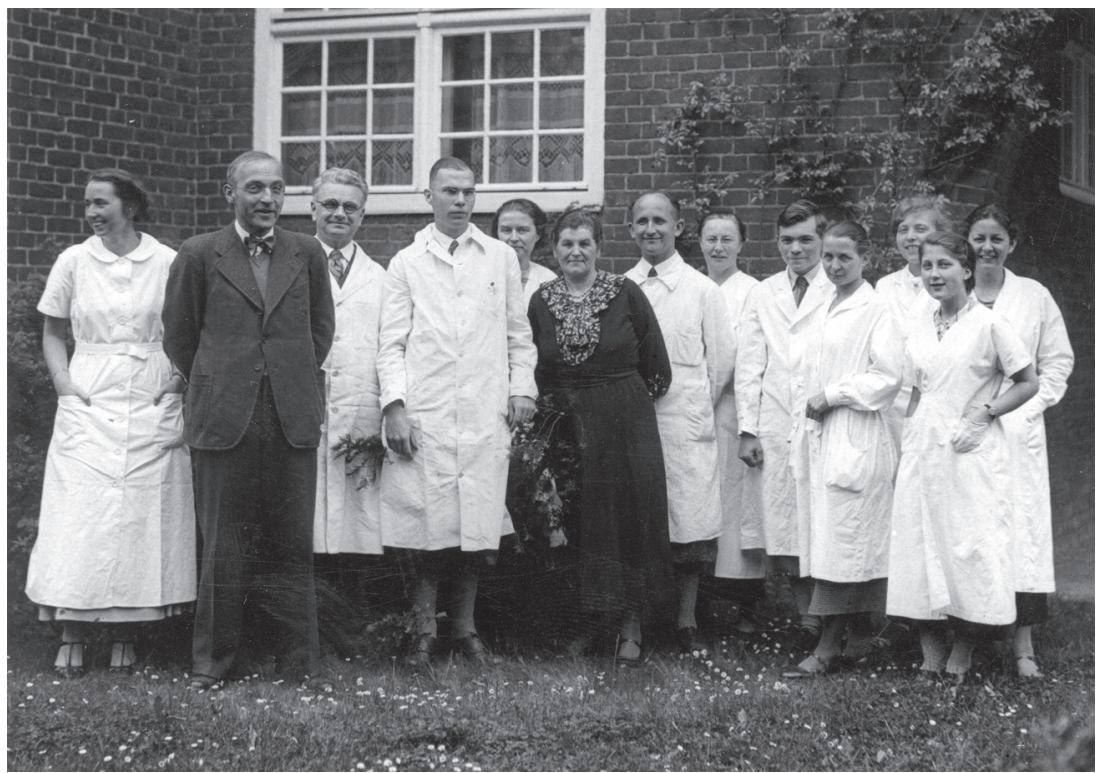

Fig. 3: Mitarbeiter des Deutschen Entomologischen Instituts zur 50-Jahrfeier 1936. Von links nach rechts: Johanna Scholze, Hans Sachtleben, Nikolai Kardakoff, Günther Schmidt, Erna Müller, Karoline Kotlarski, Richard Korschefsky, Christel Doering, Erwin Olten, Else Oelze, Maria Metzlar, Elli Kuitop, Ursula WeinRich (GR 156).

\section{Die Tradition der Larvenforschung am Deutschen Entomologischen Institut}

WiLli HenNig hatte natürlich die Bedeutung der Kenntnis der Entwicklungsstadien für phylogenetische Untersuchungen erkannt, mehr noch: eigentlich zum essentiellen Bestandteil einschlägiger Forschungen über Insekten erhoben. Aber er hatte auch die grundlegende Bedeutung der Larvenkenntnis für angewandte Fragestellungen im Sinne und förderte ihre Bestimmbarkeit. Erinnert sei an sein dreibändiges Werk über die Larvenformen der Diptera (HenNig 1948/1952).

Weniger bekannt ist, dass Willi HenNig auch den Käferlarven Aufmerksamkeit entgegen brachte, ja sogar die Reihe begründete (Hennig 1938), die vor allem durch Richard KorschefsKy bekannt geworden ist.

Es gab einen Dritten im Bunde der großen Larvenkenner: Fritz Isidor van EMDen (18981958) (Fig. 4). Er hatte von Mai 1922 bis Januar 1923 als Assistent am DEI gearbeitet. Mangelnde finanzielle Möglichkeiten gestatteten damals keine weitere Beschäftigung am Institut. Bemerkenswert ist ein Brief von Walther Horn vom 13.01.1923, in dem es heißt: „Ich wünsche dem scheidenden jungen Kollegen alles Gute mit auf den Weg und würde mich sehr freuen, wenn sich die pekuniären Verhältnisse des Entomologischen Deutschen Institutes recht bald so bessern würden, dass ich in die Lage käme, Herrn Dr. van EMDEN zu bitten, dass er wieder bei mir als Assistent eintreten könnte". Das sind Worte, die jeden erfreuen würden, aus der Feder des überaus kritischen WALTHER HoRn allerdings sind sie eine Art Adelsprädikat. 
Man kann sich kaum vorstellen, welche wissenschaftlichen Impulse aus einer gleichzeitigen und gemeinsamen Arbeit von Fritz van EMden und Richard KORSCHEFsKY am DEI in die Welt hinaus gegangen wären. Freilich hätten die politischen Verhältnisse nach 1933 auch dies zerstört. Fritz van EMden wurde in diesem Jahr aus dem Museum in Dresden entlassen und emigrierte mit seiner Familie 1936 nach London.

\section{Die Larvenreihe}

Zwischen 1938 und 1951 erschienen sechs Beiträge zur Kenntnis der Käferlarven, fünf davon von Richard KorSCHEFSKY (Tab. 1).

Die Auswahl umfasst vor allem Taxa, die als Pflanzen- oder Vorratsschädlinge einen gewissen Ruf haben. Im Deutschen Entomologischen Institut wurden auch damals viele einschlägige Anfragen aus der Praxis des Pflanzen- und

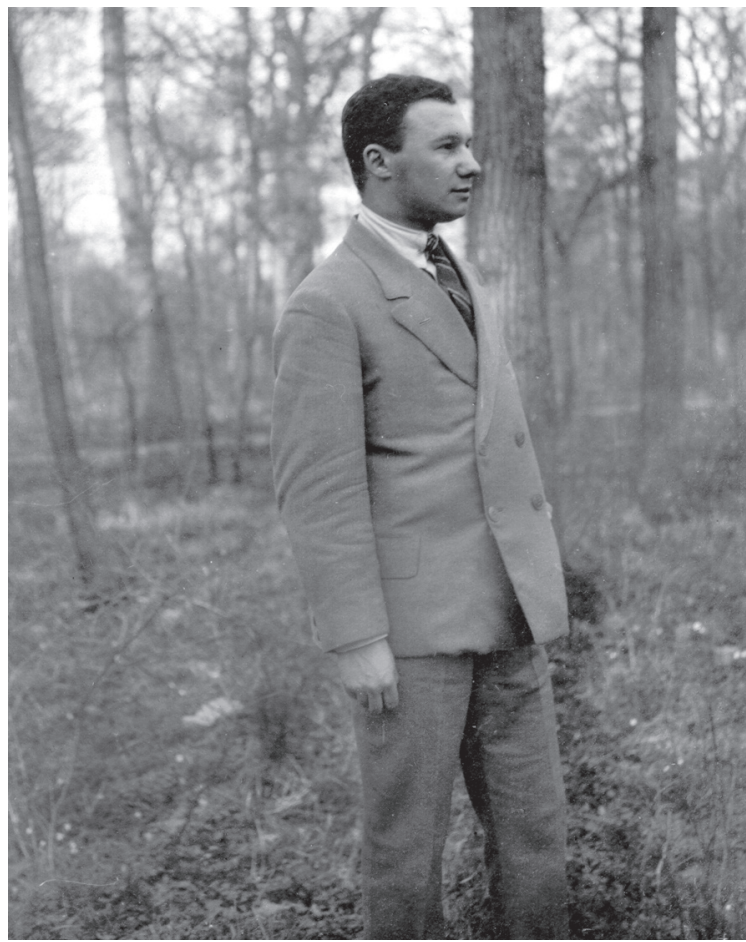

Fig. 4: Fritz IsIdor van EMden (Portraitsammlung 967).

Vorratsschutzes beantwortet. Andererseits war RICHARD KORSCHEFSKY ein begeisterter allgemeiner Sammler, wie aus seiner Sammlung zu ersehen ist (Fig. 5, 6).

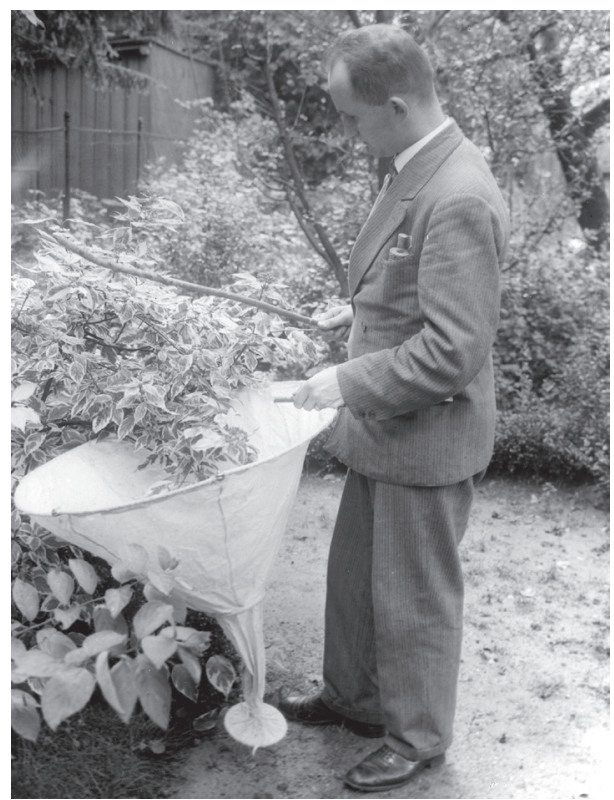

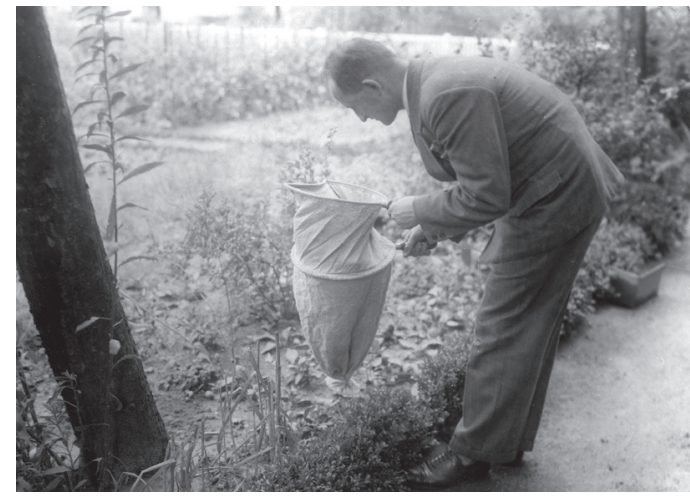

Fig. 5: (links) RICHARD KoRsCHEFSKY beim Käfersammeln im Juli 1930 (Copyright by Presse-Photo Ges. M. B. H., Berlin) (Portraitsammlung 2178).

Fig. 6: (rechts) Richard KorschefsKy mit einem Käfersieb im Juli 1930 (Copyright by Presse-Photo Ges. M. B. H., Berlin) (Portraitsammlung 2179). 
Tab. 1: Übersicht über die Bestimmungstabellen für die Larven der Coleoptera, die vom DEI herausgegeben wurden.

\begin{tabular}{|l|l|l|}
\hline Familie(n) & Jahr & Autor \\
\hline Chrysomelidae & 1938 & Hennig \\
\hline Scarabaeidae & 1940 & Korschefsky \\
\hline Elateridae & 1941 & Korschefsky \\
\hline Tenebrionidae/Alleculidae & 1943 & Korschefsky \\
\hline Dermestidae & 1944 & Korschefsky \\
\hline Lycidae, Lampyridae, Drilidae & 1951 & Korschefsky \\
\hline
\end{tabular}

Die Bestimmungstabellen sind fast ausschließlich auf dem letzten (präpupalen) Larvenstadium aufgebaut und hervorragend illustriert (Fig. 7-12). Die Abbildungen sind Originale von Hans JoHN, Berlin, nach Larvenmaterial. Diese Bestimmungstabellen bildeten über Jahrzehnte eine Basis der Kenntnis der Larven der jeweiligen Familie (Tab. 2).

Tab. 2: Vergleich der in den Bestimmungstabellen von KorschefsKy enthaltenen Gattungen (GK) und Arten (GA) mit dem gegenwärtigen Stand der Larvenkenntnis.

\begin{tabular}{|l|l|l|l|l|}
\hline Familie & GK & GA & Gattungen & Arten \\
\hline Scarabaeidae & 19 & 17 & 43 & 105 \\
\hline Geotrupidae* $^{*}$ & 2 & 2 & 6 & 8 \\
\hline Trogidae* & 1 & & 1 & 5 \\
\hline Elateridae & 22 & 34 & 48 & 128 \\
\hline Tenebrionidae & 28 & 41 & 37 & 54 \\
\hline Alleculidae** & 5 & 5 & 10 & 15 \\
\hline Dermestidae & 12 & 32 & 14 & 34 \\
\hline Omalisidae & 1 & 1 & 1 & 1 \\
\hline Lycidae & 3 & 3 & 5 & 5 \\
\hline Lampyridae & 3 & 3 & 3 & 3 \\
\hline Drilidae & 1 & 2 & 1 & 2 \\
\hline
\end{tabular}

* Geotrupidae und Trogidae sind heute selbständige Familien, früher galten sie als Unterfamilien der Scarabaeidae.

** Die Alleculidae werden heute nicht mehr als separate Familie aufgefasst, sondern als Unterfamilie der Tenebrionidae angesehen. 
Arb. physiol. angew. Ent. Berlin-Dahlem, Band 7, 1940, Nr. 1. T'afel 1.

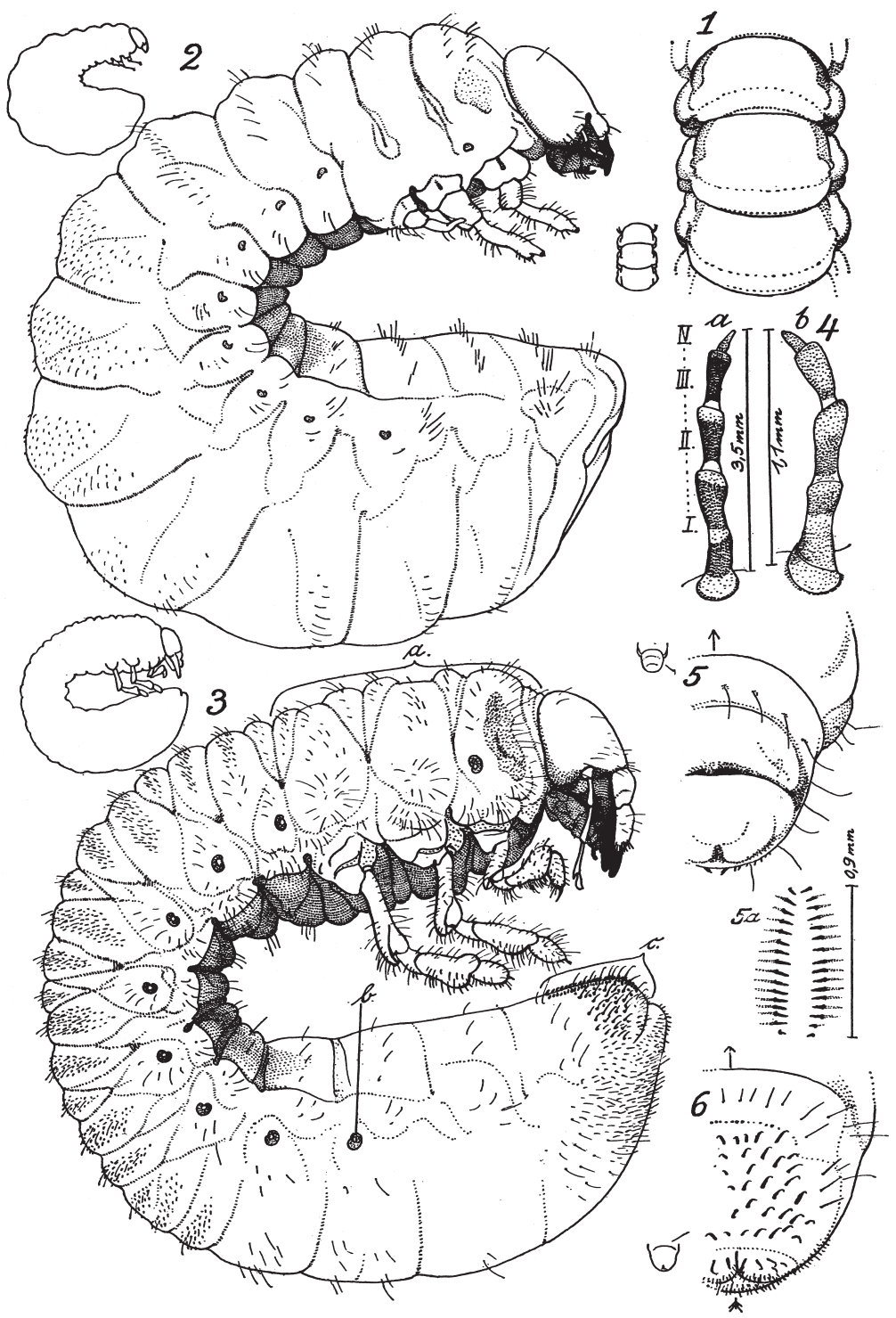

R. Korschefsky, Bestimmungstabellen der deutschen Scarabaeidenlarven.

Fig. 7: Taf. 1 aus den Bestimmungstabellen der häufigsten deutschen Scarabaeidenlarven (1940). 

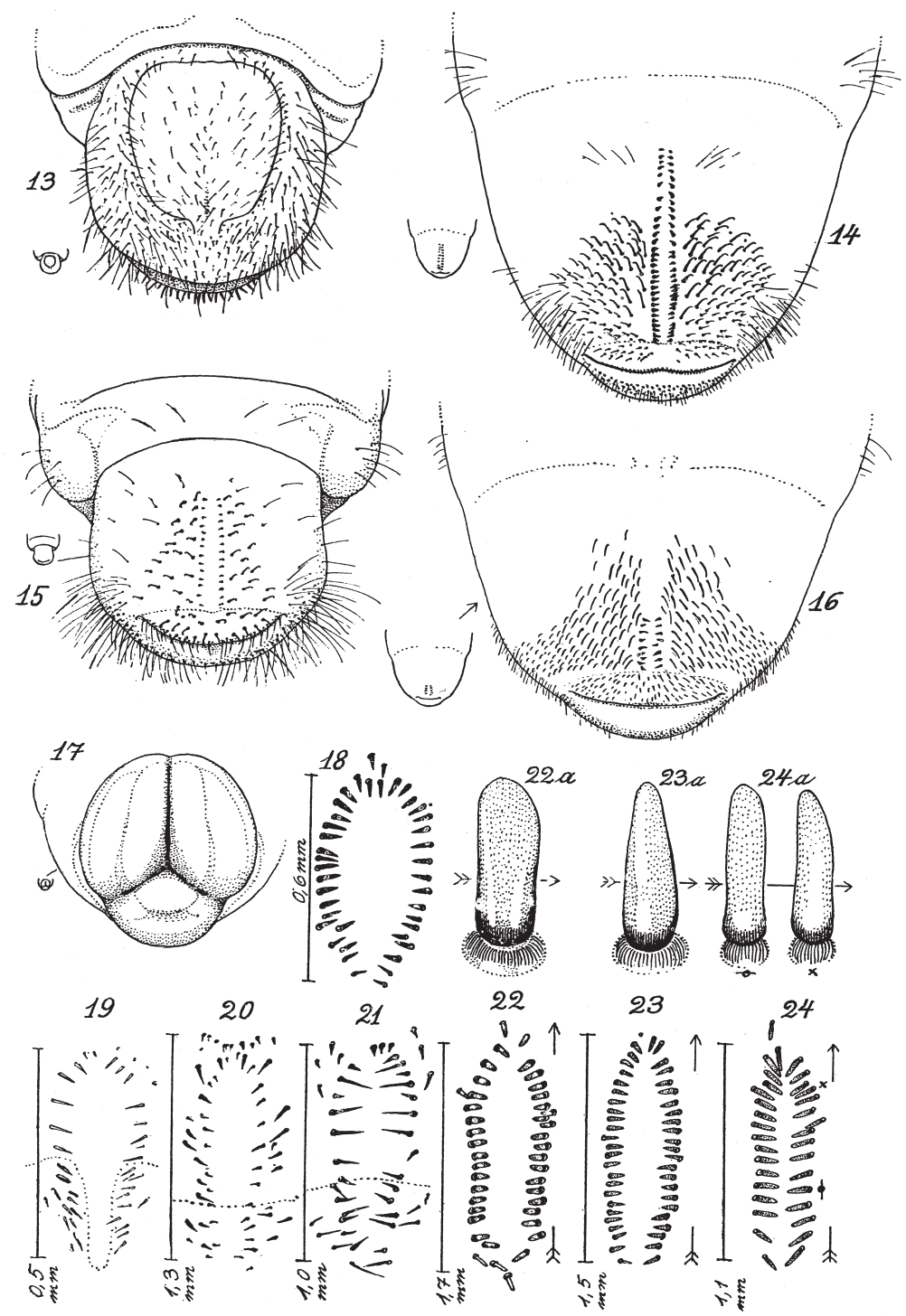

R. Korschefsky, Bestimmungstabellen der deutschen Scarabaeidenlarven.

Fig. 8: Taf. 3 aus den Bestimmungstabellen der häufigsten deutschen Scarabaeidenlarven (1940). 
Arb. morphol. taxon. Ent. Berlin-Dahlem, Band 8, 1941, Nr. 4.

Tafel 7.

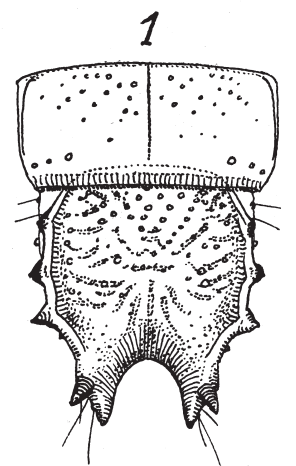

2
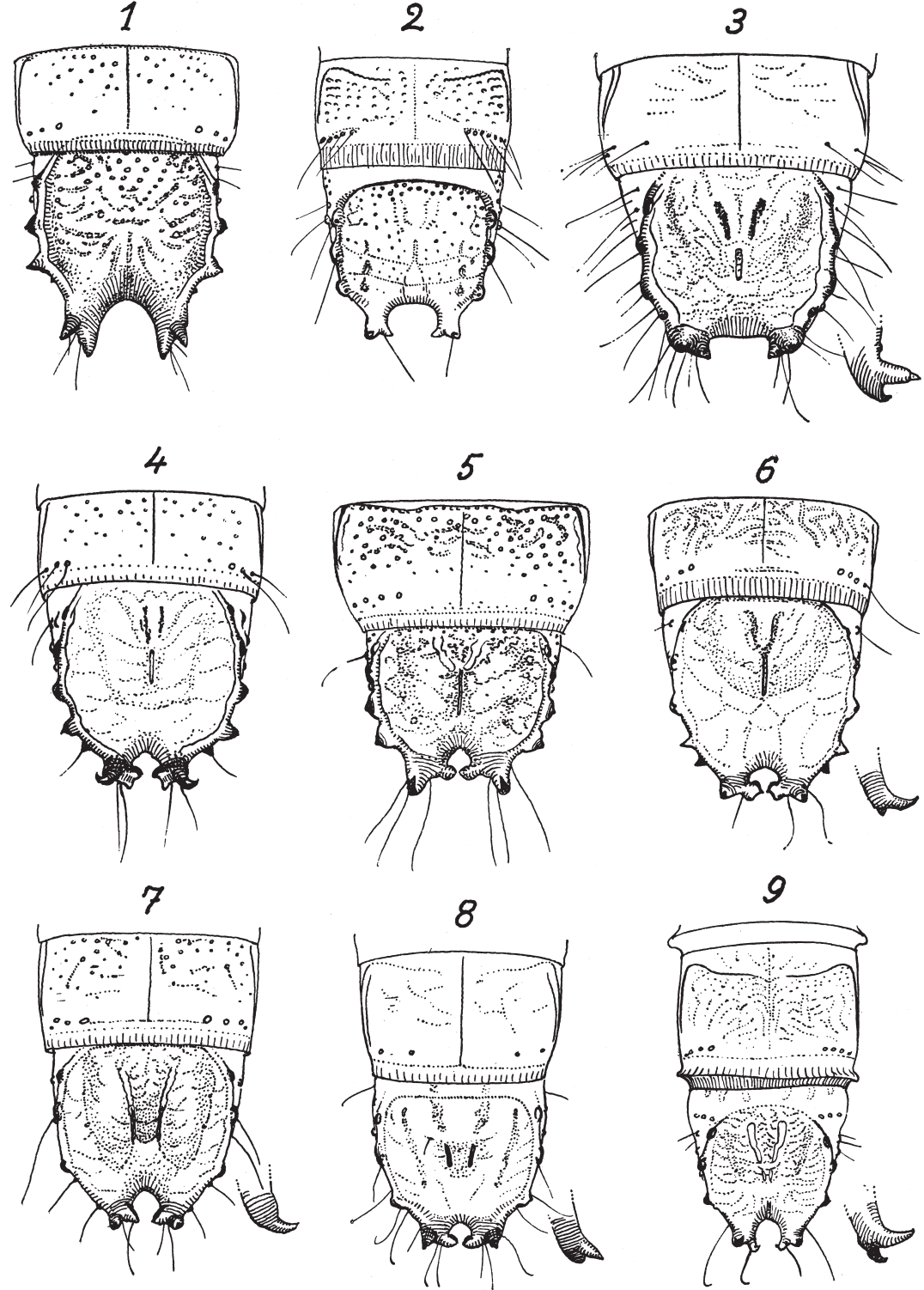

H. John del.

R. Korschefsky, Bestimmungstabelle der deutschen Elateridenlarven,

Fig. 9: Taf. 7 aus den Bestimmungstabellen der bekanntesten deutschen Elateridenlarven (1941). 


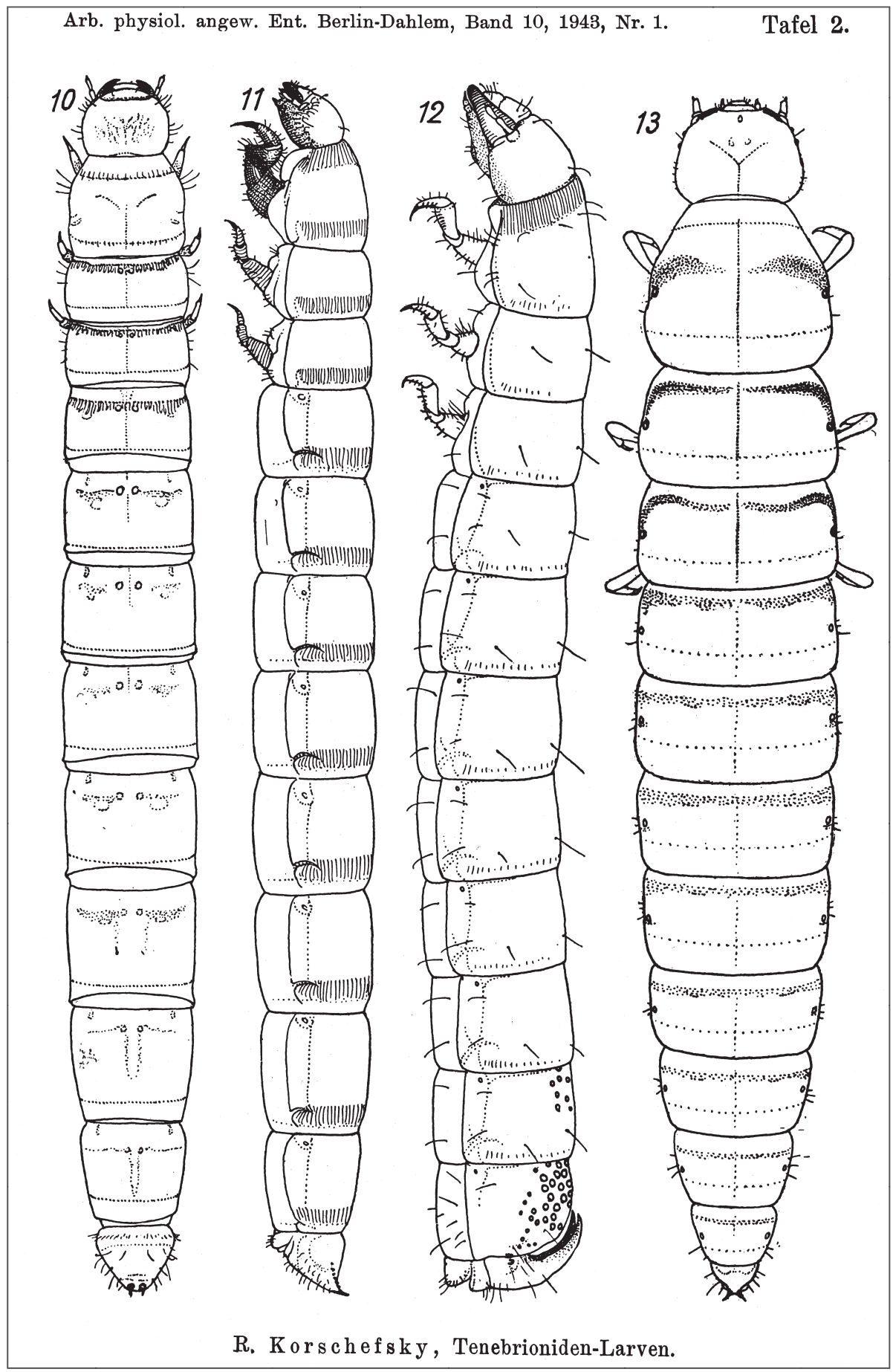

Fig. 10: Taf. 1 aus den Bestimmungstabellen der bekanntesten deutschen Tenebrioniden- und AlleculidenLarven (1943). 
Arb. physiol. angew. Ent. Berlin-Dahlem, Band 11, 1944, Nr. 2/4. Tafe] 2.

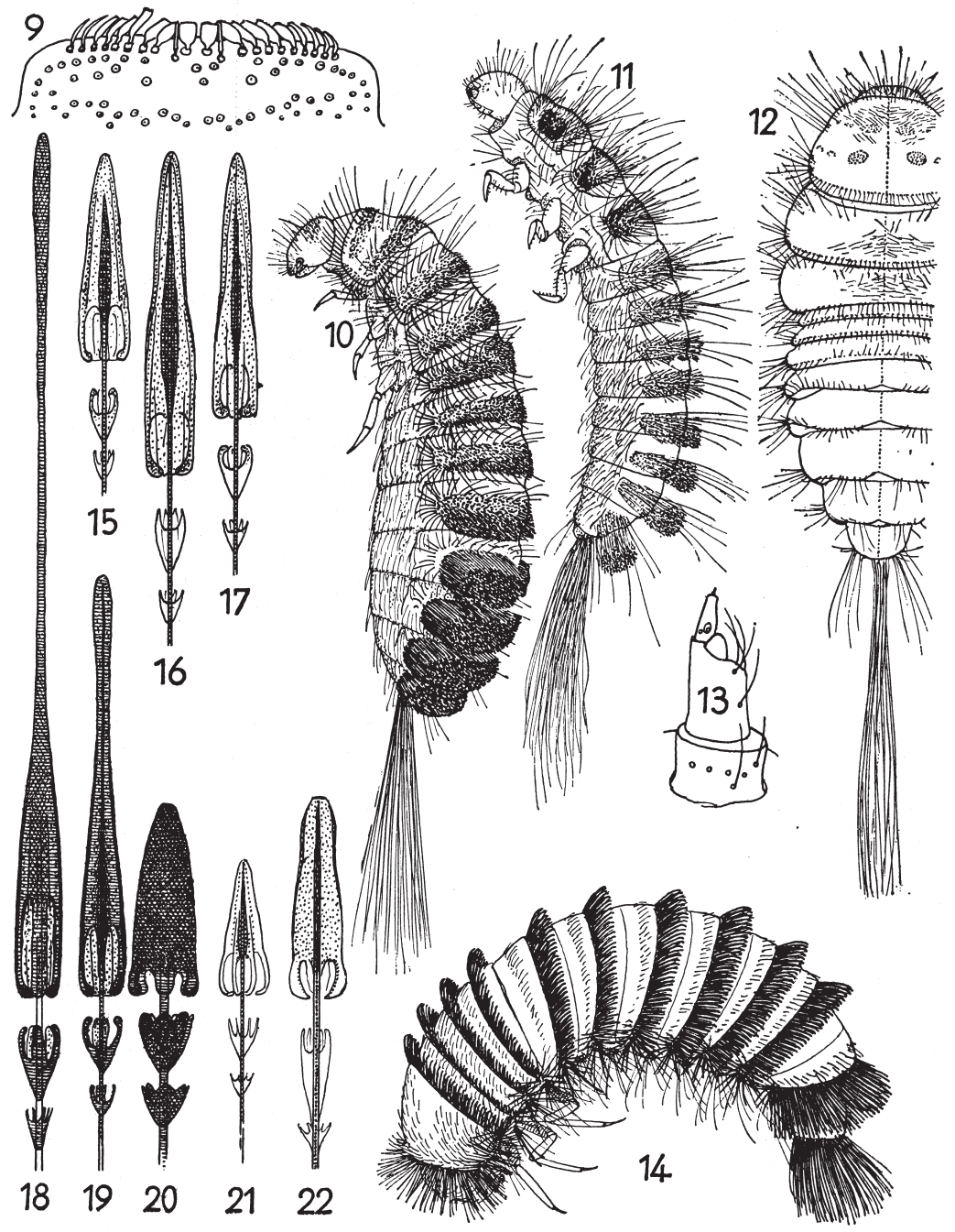

R. Korschefsky,

Bestimmungstabelle der deutschen Dermestidenlarven.

Fig. 11: Taf. 2 aus den Bestimmungstabellen der bekanntesten deutschen Dermestidenlarven (1944). 


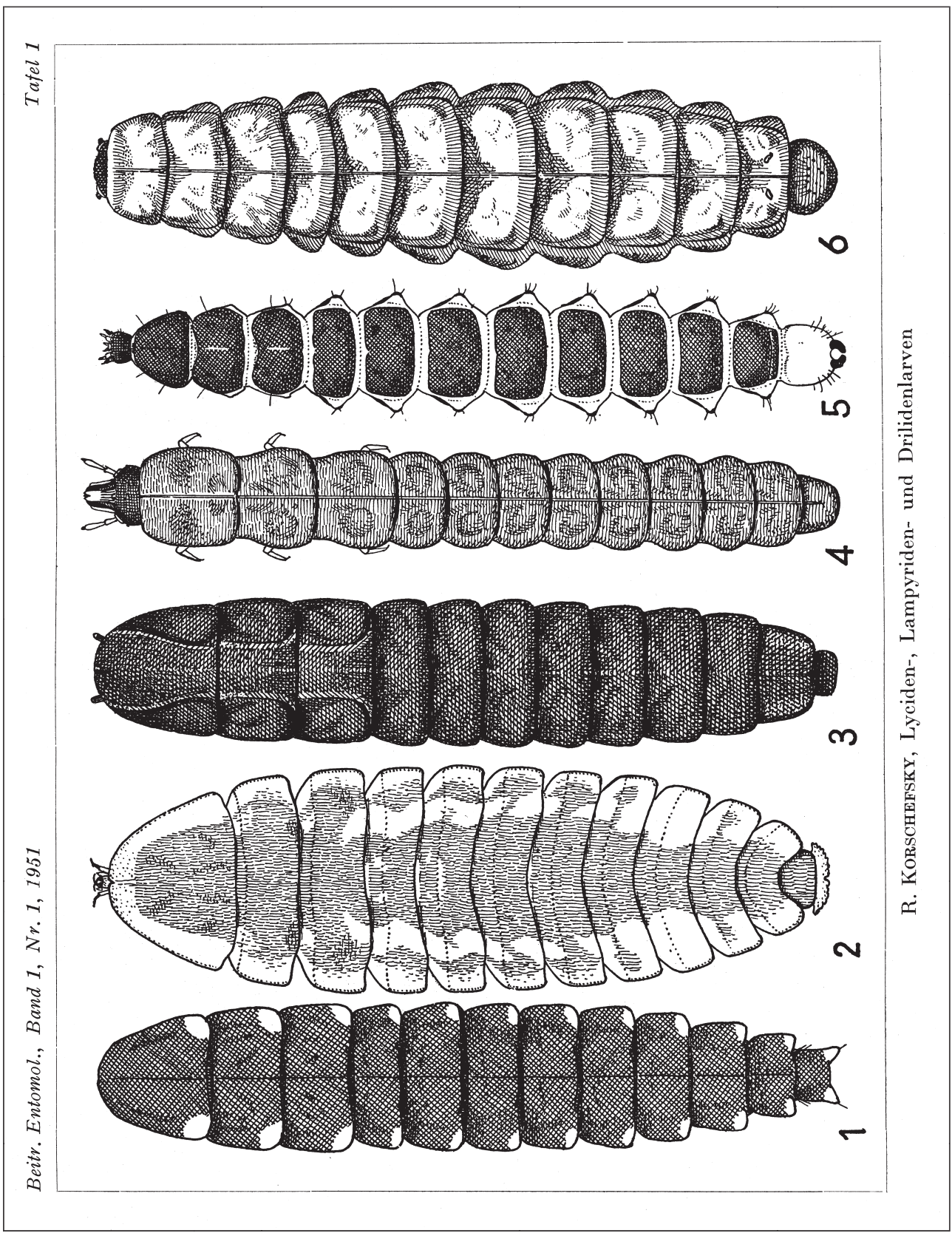

Fig. 12: Taf. 1 aus den Bestimmungstabellen der bekanntesten deutschen Lyciden-, Lampyriden- und Drilidenlarven (1951). 
Auch in der 1944 erschienenen Arbeit „Über Käfer and Käferlarven aus einem mazedonischen Weidensperlingsnest“ sind Beschreibungen von Larven enthalten (Dermestidae). In den „Arbeiten über physiologische und angewandte Entomologie aus Berlin-Dahlem“ erschien 1935 und 1936 in zwei Teilen eine Publikation über „Die Larven der Gattung Aphodius“ von Heinz Madle aus Dresden. Es ist anzunehmen, dass es eine Zusammenarbeit mit Richard Korschefsky gab.

\section{Die Liebe zu den Coccinellidae}

Warum Richard Korschefsky ausgerechnet die Coccinellidae bevorzugt bearbeitet hat, bleibt verborgen. Tatsache ist, dass er mit einer Arbeit über diese Familie seine wissenschaftliche Publikationstätigkeit begann und dass die meisten seiner Veröffentlichungen Marienkäfer zum Inhalt haben.

Nach Schmidt (1963) begann er 1925 mit einer Spezialisierung auf die Coccinellidae. Insgesamt hat Richard Korschefsky 28 Publikationen über diese Familie verfasst, vor allem über die Äthiopis, Orientalis, Neotropis und Australis, z. T. ging es um Expeditionsausbeuten.

\section{Die Bearbeitung der Coccinellidae im Junk-SchenkuIng-Katalog}

Für jeden Koleopterologen ist dieser Katalog eine wesentliche Basis. Natürlich sind die einzelnen Teile von unterschiedlicher Qualität und Aktualität. Jahrzehntelang ging und auch heute noch geht kein Weg an ihnen vorbei, auch wenn für manche Taxa und manche Regionen inzwischen wesentlich bessere Bearbeitungen vorliegen.

RICHARD Korschefsky hat in überdurchschnittlich kurzer Zeit in 2 Teilen (1931/1932) die Coccinellidae herausgebracht (Fig. 13, 14). Die weltweite Zusammenstellung führt auf 659 Seiten 3279 Arten auf und ist bis heute eine Grundlage zur Übersicht über die Weltfauna.

Er konnte dieses Werk in so hoher Qualität in nur drei Jahren seit Beginn der Publikationstätigkeit nur schreiben,

- weil er die einmalige Bibliothek des Deutschen Entomologischen Instituts zur Verfügung hatte,

- weil er über eine umfangreiche Sammlung der Coccinellidae der Welt verfügte, die sein Eigentum war (und leider 1952 an das National Museum of Natural History, Washington (USNM), verkauft wurde),

- weil er ungestört durch äußere Einflüsse arbeiten konnte (Fig. 15) und nicht ständig begründen musste, wozu sein Werk gut ist,

- und weil er in Walther Horn einen Chef hatte, der durch seine eigenen Arbeiten, seine eigene Genauigkeit Vorbild war und Anregungen zu vermitteln wusste.

So ist dieses Werk entstanden, dem man die immensen Mühen und die Ausdauer nicht ansieht. Es ist da, wirkte und wirkt in die ganze Welt.

\section{Platynaspis luteorubra}

Es gibt eine Verbindung zwischen den Coccinellidae und den Käferlarven im Werk KorsCHefsKYs: das ist Platynaspis luteorubra (Goeze, 1777). 


\section{Coleonelerorum Catallogyls}

auspiciis et auxilio

W. Junk

editus a

S. Schenkling.

Pars 118

\section{R. Korschefsky}

Coccinellidae.

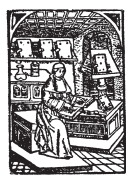

W. Junk

Berlin W 15

1931.

Fig. 13: Titelblatt der Pars 118, Coccinellidae aus dem von Junk \& Schenking herausgegebenen Coleopterorum Catalogus (1931).

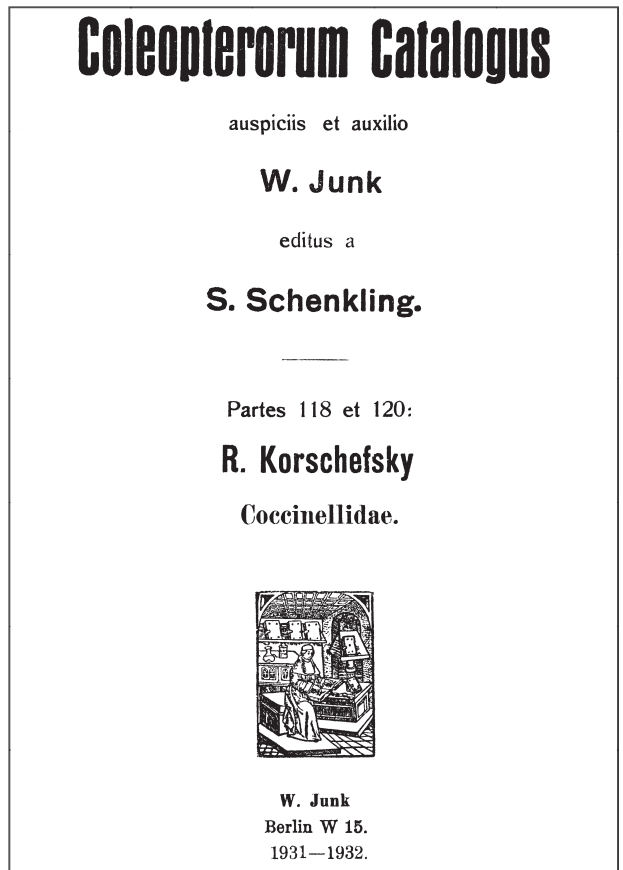

Fig. 14: Titelblatt der Partes 118 et 120, Coccinellidae aus dem von Junk \& Schenking herausgegebenen Coleopterorum Catalogus (1931/1932).

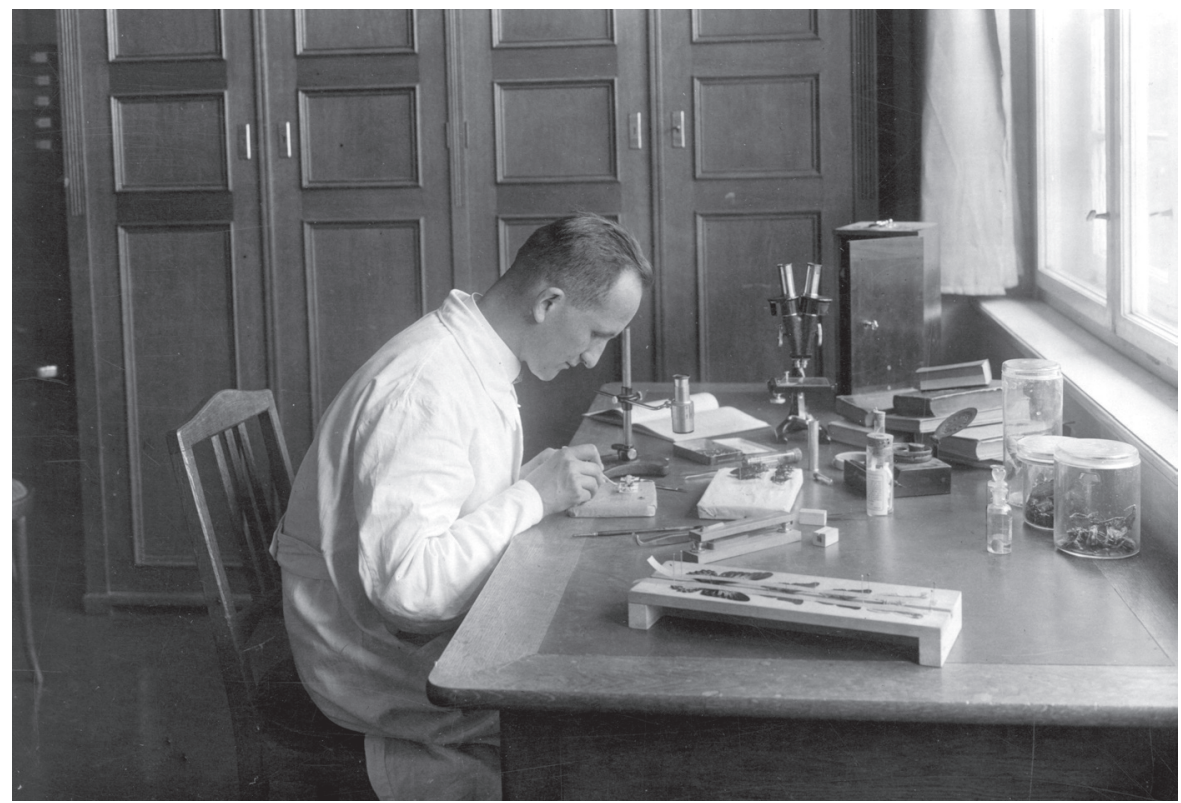

Fig. 15: Richard Korschefsky an seinem Arbeitsplatz im Juli 1930 (Foto: A. \& E. FrankL, Berlin) (Portraitsammlung 2176). 
Arb. phys. angew. Ent. Berlin-Dahlem, Band I, 1934, Nr. $4 . \quad$ Tafel 4
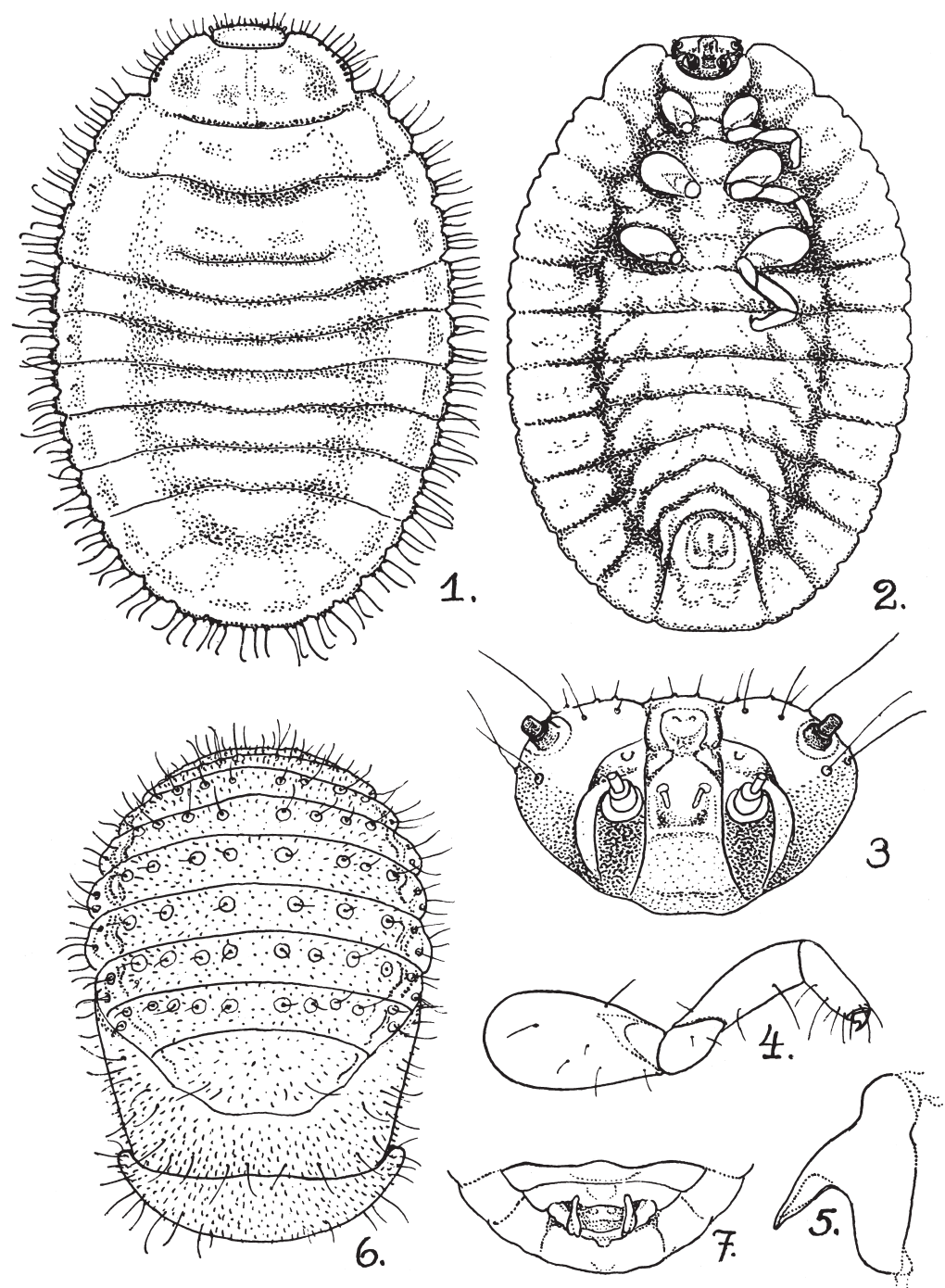

R. K orschefsky, Platynaspis luteorubra Goeze (H. JoHN del.).

Fig. 1 Larve dorsal

Fig. 2 La ve ventral

Fig 3 Larvenkopf
Fig. 4 Larvenbein

Fig. 5 Larvenmandibel

Fig. 6 Puppe

Fig. 7 Letzte Abdominalsegmente der Larve.

Fig. 16: Taf. 4 aus „Platynaspis luteorubra Goeze, ein neuer Larventypus der Coccinelliden“ (1934). 
Der Körperbau der Larven der Coccinellidae ist natürlich von Taxon zu Taxon unterschiedlich, zum Glück, sonst könnten wir die einzelnen Arten nicht nach äußeren Merkmalen trennen. Aber alle Larven ähneln sich doch und es gibt einen Habitus, der für die gesamte Familie typisch ist (wenn man die mitteleuropäischen Arten betrachtet).

Eine wesentliche Ausnahme liegt vor, das ist Platynaspis luteorubra, deren Larven einen völlig anderen Habitus haben und die man unvorbereitet keinesfalls als Coccinellidenlarven ansprechen würde. RICHARD KORSCHEFSKY hat diese Larven gefunden, durch Aufzucht ihre Art erkannt und beschrieben (1934 als erste seiner Larvenarbeiten) (Fig. 16). Heute ist es koleopterologisches Allgemeinwissen.

\section{Schlussgedanken}

Der Blick auf das Werk von Richard KorsChefsKy sollte nachdenklich stimmen, auch weil es in gewissem Sinne nur ein Beispiel ist. RichaRd KorsCHEFSKY ist jung gestorben, wir haben nur ein Fragment des Möglichen (44 Publikationen).

Er kam durch den Krieg um, wie so viele, damals und noch immer.

Er war eine Begabung, ein Talent, wurde gefördert unabhängig eines akademischen Abschlusses - das kann auch heute so sein.

Er machte etwas aus den Arbeitsmöglichkeiten dieses Hauses - das kann auch heute so sein.

Er durfte arbeiten, seine Themen, seine für das DEI typischen Themen, wurden nicht in Frage gestellt - das kann auch heute so sein.

RichARD KorscheFsKYs Werk wurde nicht nach ephemeren Kriterien bemessen, es wirkt noch nach 7 Jahrzehnten in der ganzen Welt - das kann auch heute so sein.

„Der Blick in die Vergangenheit erhellt uns die Gegenwart und weist uns die Zukunft“.

\section{Verzeichnis der Schriften von RichaRD KorschefsKY}

Korschefsky, R. 1928: Bemerkungen über exotische Coccinellidae der alten Welt mit Beschreibung einer neuen Art. (1. Beitrag zur Kenntnis der Coccinelliden). - Entomologische Mitteilungen. Herausgegeben vom Verein zur Förderung des Deutschen Entomologischen Museums 17: 41-43, 8 figs.

KorschefsKy, R. 1928: Bemerkungen über afrikanische Epilachninen mit Beschreibung zweier neuer Arten (Coleopt.). (2. Beitrag zur Kenntnis der Coccinelliden). - Wiener Entomologische Zeitung 45: 122-125, 3 figs.

Korschefsky, R. 1928: Die entomologischen Publikationen von Julius Weise. - Entomologische Blätter 28: $175-186$.

KorschefsKy, R. 1929: Bemerkungen über afrikanische Epilachninen mit Beschreibung einer neuen Art (Col.). - Deutsche Entomologische Zeitschrift 1929: 141-143, 3 figs.

Korschefsky, R. 1929: Synonymische und andere Bemerkungen zum Genus Caria Mulsant - Ailocaria Свотсн. - Zoologischer Anzeiger 82: 86-89, 7 figs.

Korschefsky, R. 1931: Pars 118, Coccinellidae. - In: Junk, W. \& Schenkuing, S. (Hrsg.): Coleopterorum Catalogus: 1-224; Berlin.

Korschefsky, R. 1931/1932: Partes 118 et 120, Coccinellidae. - In: Junk, W. \& Schenkling, S. (Hrsg.): Coleopterorum Catalogus: 225-659; Berlin.

Korschefsky, R. 1933: Bemerkungen über Coccinelliden von Formosa. - Transactions of the Natural History Society of Formosa 23, nos. 128 \& 129: 299-304, 17 figs.

Korschefsky, R. 1933: Synonymische und andere Bemerkungen über Свотсн'sche Coccinelliden-Typen. - Stylops: A Journal of Taxonomie Entomology 2, Part 10: 236-237.

Korschefsky, R. 1934: Bemerkungen über Coccinelliden und Beschreibung einer neuen Neda-Art (Col.). - Arbeiten über morphologische und taxonomische Entomologie aus Berlin-Dahlem 1: 267-271.

Korschefsky, R. 1934: Drei neue Coccinelliden der Indomalayischen and papuanischen Region. - Mitteilungen der Schweizerischen Entomologischen Gesellschaft 16 (2): 107-109, 2 Textfig. 
Korschefsky, R. 1934: Entomological investigations on the spike disease of Sandal 16. Coccinellidae (Col.). - Indian Forest Records 19, Part 6: 1-10, 1 Taf.

Korschefsky, R. 1934: Platynaspis luteorubra Goeze, ein neuer Larventypus der Coccinelliden. - Arbeiten über physiologische und angewandte Entomologie aus Berlin-Dahlem 1: 278-279, Taf. 4.

Korschefsky, R. 1934: Prof. Dr. E. Handschin, Studienreise auf den Sundainseln und in Nordaustralien, 1930-32. 3. Drei neue Coccinelliden der Indomalayischen und papuanischen Region. - Mitteilungen der Schweizerischen Entomologischen Gesellschaft 16: 107-109, 2 figs.

Korschefsкy, R. 1934: Weitere Bemerkungen über Свотсн'sche Typen und Beschreibung einer neuen Oryssomus-Art aus dem Deutschen Entomologischen Institut. - Arbeiten über morphologische und taxonomische Entomologie aus Berlin-Dahlem 1: 35-36.

Korschefsky, R. 1935: Coccinellidae. - In: Visser, P. C. \& Visser-Hooft, J. (Hrsg.): Wissenschaftliche Ergebnisse der Niederländischen Expedition in die Karakorum und die angrenzenden Gebiete 1922, 1925 und 1929-1930. - Verlag F. A. Brockhaus, Leipzig, 1: 299, 1 Fig.

Korschefsky, R. 1935: Entomological expedition to Abyssinia, 1926-27. Coleoptera, Coccinellidae. With introduction and notes by Hugh Sсотт. - Annals and Magazine of Natural History, London (10) 15: 53-65, 6 figs.

Korschefsky, R. 1935: Neue Coccinelliden aus Afrika, Brasilien und Formosa. - Arbeiten über morphologische und taxonomische Entomologie aus Berlin-Dahlem 2: 252-256, 5 figs.

Korschefsky, R. 1935: Ueber die Coleopteren-Ausbeute der II. Schweizer wissenschaftlichen Expedition nach Angola 1932-33. III. Coccinelliden. - Stettiner entomologische Zeitung 96: 169-170, 3 figs.

Korschefsky, R. 1936: Eine neue Cryptognatha-Art aus Süd-Amerika (Coleoptera: Coccinellidae). Arbeiten über morphologische und taxonomische Entomologie aus Berlin-Dahlem 3: 299-300.

Korschefsky, R. 1936: Zum fünfzigjährigen Bestehen des Deutschen Entomologischen Institutes der Kaiser-Wilhelm-Gesellschaft, Berlin-Dahlem. - Entomologische Blätter 32: 89-92, 1 Taf.

Korschefsky, R. 1937: Zwei neue Novius-Arten von den Canaren. (9. Beitrag zur Kenntnis der Coccinellidae.). - Societas Scientiarum Fennica, Commentationes Biologicae 6 (3): 1-3, 2 figs.

Korschefsky, R. 1937: Beobachtungen an Meloe violaceus L. und Notoxus monoceros L. - Arbeiten über physiologische und angewandte Entomologie aus Berlin-Dahlem 4: 157-158.

KoRsChefsky, R. 1937: Entomologische Sammelergebnisse der Deutschen Hindukusch-Expedition 1935 der Deutschen Forschungsgemeinschaft. Coccinellidae. - Arbeiten über morphologische und taxonomische Entomologie aus Berlin-Dahlem 4: 182-183.

Korschefsky, R. 1938: Eine neue Cycloneda-Art aus Brasilien (Coleoptera: Coccinellidae). - Arbeiten über morphologische und taxonomische Entomologie aus Berlin-Dahlem 5: 264.

Korschefsky, R. 1938: Synonymische und andere Bemerkungen über Coccinelliden und Beschreibung einer neuen Pristonema-Art. - Entomologische Blätter 34: 40-41, 1 fig.

Korschefsky, R. 1939: Abbildungen und Bemerkungen zu vier Schaufuss'schen Coleopteren aus dem deutschen Bernstein. - Arbeiten über morphologische und taxonomische Entomologie aus BerlinDahlem 6: 11-12, Taf. 1.

Korschefsky, R. 1939: Dr. Walther Horn †. - Entomologische Blätter 35: 177-184.

Korschefsky, R. 1939: Zur Erinnerung an Dr. Walther Horn. - Entomologische Rundschau mit Societas entomologica 56: 385-387.

KorschefsKy, R. 1940: Bestimmungstabelle der häufigsten deutschen Scarabaeidenlarven. - Arbeiten über physiologische und angewandte Entomologie aus Berlin-Dahlem 7: 41-52, Taf. 1-3.

KorschefsKy, R. 1940: Über Coccinellidae von Erythrea. - Redia 26: 71-72.

KoRsCHEFSKY, R. 1940: Verzeichnis der Sammelindices von Zeitschriften mit mehr oder weniger entomologischem Inhalt. - Arbeiten über morphologische und taxonomische Entomologie aus Berlin-Dahlem 7: 209-225.

Korschefsky, R. 1940: Vier neue Coccinelliden der Alten Welt. - Entomologische Blätter 36: 1-3. 
Korschefsky, R. 1941: Bestimmungstabelle der bekanntesten deutschen Elateridenlarven (Coleoptera: Elateridae). - Arbeiten über morphologische und angewandte Entomologie aus Berlin-Dahlem 8: 217230, Taf. 5-7.

Korschefsky, R. 1941: Die historischen Exemplare fremder Autoren in STIERLIN's Coleopteren-Sammlung. - Arbeiten über morphologische und taxonomische Entomologie aus Berlin-Dahlem 8: 267-285, 9 Fig.

Korschefsky, R. 1942: Sachverzeichnis der „Entomologische Blätter“ Jahrgang 1, 1905 bis 37, 1941. Entomologische Blätter 38: 1-112.

Korschefsky, R. 1943: Bestimmungstabelle der bekanntesten deutschen Tenebrioniden- und AlleculidenLarven (Coleoptera). - Arbeiten über physiologische und angewandte Entomologie aus Berlin-Dahlem 10: 58-68, Taf. 1-4.

Korschefsky, R. 1943: Ueber Coccinelliden von den Neuen Hebriden aus dem National-Museum, Paris. - Entomologische Blätter 39: 51-52, 3 figs.

Korschefsky, R. 1944: Bestimmungstabelle der bekanntesten deutschen Dermestidenlarven (Coleoptera). - Arbeiten über physiologische und angewandte Entomologie aus Berlin-Dahlem 11: 140-152, Taf. 1-2.

Korschefsky, R. 1944: Kritische Bemerkungen über Coccinellidentypen von Theodor Kirsch mit Beschreibung einer neuen Art und Form. - Entomologische Blätter 40: 133-137.

KorschefsKy, R. 1944: Neue altweltliche Coccinelliden (Coleoptera: Coccinellidae). - Arbeiten über morphologische und taxonomische Entomologie aus Berlin-Dahlem 11: 47-56, 6 Fig.

Korschefsky, R. 1944: Über Käfer and Käferlarven aus einem mazedonischen Weidensperlingsnest (Coleoptera: Tenebrionidae \& Dermestidae). - Arbeiten über morphologische und taxonomische Entomologie aus Berlin-Dahlem 11: 77-80, 4 Fig.

Korschefsky, R. 1944: Über fünf bisher nicht gedeutete deutsche Coleopterenbeschreibungen aus dem Jahre 1799. - Entomologische Blätter 40: 27-29.

KorsChefsky, R. 1951: Bestimmungstabelle der bekanntesten deutschen Lyciden-, Lampyriden- und Drilidenlarven (Coleoptera). - Beiträge zur Entomologie 1: 60-64, Taf. 1.

Es ist nicht sicher, ob dieses Verzeichnis vollständig ist, Ergänzungen sind sehr willkommen.

\section{Dank}

Ohne die Hilfe und Unterstützung durch den Leiter des Deutschen Entomologischen Instituts, Herrn Prof. Dr. Holger H. Dathe und Herrn Christian Kutzscher wäre diese Hommage an Richard Korschefsky nicht möglich gewesen, herzlichen Dank!

\section{Literatur}

Evers, A. M. J. 1974: 70 Jahre Entomologische Blätter. Ein Beitrag zur Entomo-Bibliographie und Geschichte der Entomologie. Rückblick und Ausschau. - Entomologische Blätter 70 (3): 129-145.

Hennig, W. 1938: Übersicht über die Larven der wichtigsten deutschen Chrysomelinen. - Arbeiten über physiologische und angewandte Entomologie aus Berlin-Dahlem 5: 85-136.

Hennig, W. 1948/1952: Die Larvenformen der Dipteren. Teil 1-3. - Akademie Verlag, Berlin, 1270 S.

Madle, H. 1935/1936: Die Larven der Gattung Aphodius I. - Arbeiten über physiologische und angewandte Entomologie aus Berlin-Dahlem 2: 289-304, 3: 1-20.

Schmidt, G. 1963: Richard KorschefsKy zum Gedenken. - Entomologische Blätter 59 (3): 129-131.

Anschrift des Verfassers:

Prof. Dr. Bernhard Klausnitzer

Mitglied des DEI

Lannerstraße 5

01219 Dresden, Deutschland

\section{Subject editor:}

Prof. Dr. H. H. Dathe 